\title{
MÖBIUS INVERSION FORMULAE FOR APOSTOL-BERNOULLI TYPE POLYNOMIALS AND NUMBERS
}

\author{
A. BAYAD AND J. CHIKHI
}

\begin{abstract}
In this paper, we establish Möbius inversion formulae for the Fourier expansions of the Apostol-Bernoulli, Apostol-Euler and ApostolGenocchi polynomials. As an application, by specializing our formulae at some special values we obtain interesting number-theoritical relations. We derive explicit formulae for Apostol-Bernoulli numbers. These formulae involve Stirling numbers of the second kind and powers of cotangent. Our proofs are very simple.
\end{abstract}

\section{INTRODUCTION}

For $\omega \in \mathbb{C} \backslash\{0\}$ and $x$ variable, the $n^{\text {th }}$ Apostol-Bernoulli polynomial $B_{n}(x ; \omega)$ is defined by the generating function

$$
\sum_{n \geq 0} B_{n}(x ; \omega) \frac{t^{n}}{n !}=\frac{t e^{x t}}{\omega e^{t}-1},(|t+\log (\omega)|<2 \pi),
$$

where

$$
\omega=|\omega| e^{i \theta},-\pi \leq \theta<\pi \text { and } \log (\omega)=\log |\omega|+i \theta .
$$

For $\omega=1, B_{n}(x ; 1)=B_{n}(x)$ is the $n^{\text {th }}$ Bernoulli polynomial and $B_{n}(0 ; 1)=B_{n}$ is the $n^{\text {th }}$ Bernoulli number.

The Möbius inversion formula for the Bernoulli polynomials $B_{n}(x)$ has been studied in $[5]$ for $n \geq 2$. Hence, the purpose of this paper is to investigate the case $\omega \neq 1$.

It is well known that the Apostol-Bernoulli polynomials (see [2] or [4] ) has the following Fourier expansion,

$$
-\frac{\omega^{x}}{n !} B_{n}(x ; \omega)=\sum_{k \in \mathbb{Z}} \frac{e^{2 i \pi k x}}{(2 i \pi k-\log (\omega))^{n}},
$$

for $0<x<1$ if $n=1,0 \leq x \leq 1$ if $n \geq 2$.

Remark 1. The convergence, of the Fourier series (3), is absolute and uniform in $[0,1]$ except for $B_{1}(x ; \omega)$. In this paper, the case $n=1$ is not treated.

The paper is organized as follows. In section 2, we establish a Möbius inversion type formula for Apostol-Bernoulli polynomials. For that, we shall slightly modify

Received by the editor November 20, 2011 and, in revised form, January 25, 2012.

2010 Mathematics Subject Classification. Primary 11B68, 11A25, 11B73, 42A16, 41A58.

Key words and phrases. Apostol-Bernoulli numbers and polynomials, Apostol-Euler polynomials, Apostol-Genocchi polynomials, Stirling numbers, Fourier series, Möbius function, Möbius inversion. 
the expression (3). Similarly, in section 3, we obtain Möbius inversion formulae for Apostol-Euler and Apostol-Genocchi polynomials.

\section{Möbius Inversion for Apostol-Bernoulli POlynomials}

With a view towards stating our main results, we transform the Fourier expansion (3) in sum over positive integers. Let us define a 1-periodic, in the variable $x$, function by

$$
F_{n}(x ; \omega)=-\frac{\omega^{x}}{n !} B_{n}(x ; \omega)-\frac{1}{(-\log (\omega))^{n}},
$$

for $0<x<1$ if $n=1$, and $0 \leq x \leq 1$ if $n \geq 2$.

We set

$$
f_{n}(k ; x ; \omega)=\frac{e^{2 i \pi k x}}{(2 i \pi k-\log (\omega))^{n}}+\frac{e^{-2 i \pi k x}}{(-2 i \pi k-\log (\omega))^{n}},
$$

then from (3) we observe that

$$
F_{n}(x ; \omega)=\sum_{k=1}^{\infty} f_{n}(k ; x ; \omega),
$$

and its Möbius inversion is given by the following interesting and useful theorem.

Theorem 1. For any positive integer $n \geq 2$, real $x$ and complex $\omega \in \mathbb{C} \backslash\{0,1\}$, we have

$$
f_{n}(1 ; x ; \omega)=\sum_{m=1}^{\infty} \frac{\mu(m)}{m^{n}} F_{n}\left(m x ; \omega^{1 / m}\right),
$$

where $\mu$ is the arithmetical Möbius function.

Proof. We first note that the function $f_{n}$ satisfies the remarkable property,

$f_{n}\left(k ; m x ; \omega^{1 / m}\right)=\frac{e^{2 i \pi m k x}}{(2 i \pi k-\log (\omega) / m)^{n}}+\frac{e^{-2 i \pi m k x}}{(-2 i \pi k-\log (\omega) / m)^{n}}=m^{n} f_{n}(m k ; x ; \omega)$.

Hence for any integer $n \geq 2$, real $x$ and complex $\omega \in \mathbb{C} \backslash\{0,1\}$, we have

$$
\begin{aligned}
& \sum_{m=1}^{\infty} \sum_{k=1}^{\infty}\left|\frac{\mu(m)}{m^{n}} f_{n}\left(k ; m x ; \omega^{1 / m}\right)\right| \\
& \quad \leq \sum_{m=1}^{\infty} \sum_{k=1}^{\infty}|\mu(m)|\left(\frac{1}{|2 i m k \pi-\log (\omega)|^{n}}+\frac{1}{|2 i m k \pi+\log (\omega)|^{n}}\right) .
\end{aligned}
$$

Using the elementary inequalities,

$$
\begin{aligned}
& |2 i m k \pi \pm \log (\omega)| \geq 2 m k \pi-|\arg (\omega)| \geq 2 m k \pi-\pi \geq m k \pi, \\
& |\mu(m)| \leq 1,(\forall m \geq 1),
\end{aligned}
$$

we obtain

$$
\sum_{m=1}^{\infty} \sum_{k=1}^{\infty}\left|\frac{\mu(m)}{m^{n}} f_{n}\left(k ; m x ; \omega^{1 / m}\right)\right| \leq \frac{2}{\pi^{n}} \sum_{m=1}^{\infty} \sum_{k=1}^{\infty} \frac{1}{m^{n} k^{n}} \leq \frac{2}{\pi^{n}} \zeta^{2}(n) .
$$


The convergence is then absolute and uniform. Substituting the expansion (4) of $F_{n}$ in the right-hand of (5), we obtain

$$
\begin{aligned}
\sum_{m=1}^{\infty} \frac{\mu(m)}{m^{n}} F_{n}\left(m x ; \omega^{1 / m}\right) & =\sum_{m=1}^{\infty} \sum_{k=1}^{\infty} \frac{\mu(m)}{m^{n}} f_{n}\left(k ; m x ; \omega^{1 / m}\right)=\sum_{m, k=1}^{\infty} \mu(m) f_{n}(m k ; x ; \omega) \\
& =\sum_{m=1}^{\infty}\left(\sum_{d \mid m} \mu(d)\right) f_{n}(m ; x ; \omega) .
\end{aligned}
$$

To end the proof of the theorem we use the following analytical-number property: for every positive integer $m$ we have

$$
\sum_{d \mid m} \mu(d)=\left\{\begin{array}{lll}
1 & \text { if } & m=1 \\
0 & \text { if } & m \geq 2
\end{array}\right.
$$

Remark 2. Theorem 1 seems to fit into the general framework for the Möbius inversion outlined in [3]. In fact, we observe that Fourier series (3) has the form

$$
F_{n}(x ; \omega)=\sum_{k \geq 1} \alpha(k) f_{n}(\phi(k ; x, \omega)),
$$

where the $\alpha(k)=k^{-n}$ is a completely multiplicative arithmetical function, and $\phi:(k ; x, \omega) \mapsto\left(k x, \omega^{k}\right)$ is a flow, i.e., $\phi(m ; \phi(k ; x, \omega))=\phi(m k ; x, \omega)$.

Now we can rewrite the Theorem 1 in terms of Apostol-Bernoulli polynomials.

Theorem 2. Let $\omega \in \mathbb{C} \backslash\{0,1\}, x$ a real number and $n \geq 2$ an integer. Then we have

$$
\begin{aligned}
& \frac{e^{2 i \pi x}}{(2 i \pi-\log (\omega))^{n}}+\frac{e^{-2 i \pi x}}{(-2 i \pi-\log (\omega))^{n}} \\
& \quad=-\sum_{m=1}^{\infty} \frac{\mu(m)}{m^{n}}\left(\frac{\omega^{\frac{\{p x\}}{m}}}{n !} B_{n}\left(\{m x\} ; \omega^{1 / m}\right)+\frac{m^{n}}{(-\log (\omega))^{n}}\right),
\end{aligned}
$$

where $\{m x\}$ denote the fractional part of $m x$.

\section{The Apostol-Euler and Apostol-Genocchi polynomials}

For $\omega \in \mathbb{C} \backslash\{0\}$ and $x$ variable, the Apostol-Euler polynomial $E_{n}(x ; \omega)$ and the Apostol-Genocchi polynomial $G_{n}(x ; \omega)$ are given by the generating functions

$$
\begin{aligned}
& \sum_{n \geq 0} E_{n}(x ; \omega) \frac{t^{n}}{n !}=\frac{2 e^{x t}}{\omega e^{t}+1},(|t+\log (-\omega)|<2 \pi), \\
& \sum_{n \geq 0} G_{n}(x ; \omega) \frac{t^{n}}{n !}=\frac{2 t e^{x t}}{\omega e^{t}+1},(|t+\log (-\omega)|<2 \pi) .
\end{aligned}
$$

They also have Fourier type expansions (see [2]). In fact, for any complex $\omega \in$ $\mathbb{C} \backslash\{-1,0\}$, we have

$$
\frac{\omega^{x}}{n !} E_{n}(x ; \omega)=2 \sum_{k \in \mathbb{Z}} \frac{e^{2 i \pi\left(k+\frac{1}{2}\right) x}}{\left(2 i \pi\left(k+\frac{1}{2}\right)-\log (\omega)\right)^{n+1}},
$$


for $0<x<1$ if $n=0$ and $0 \leq x \leq 1$ if $n \geq 1$, and

$$
\frac{\omega^{x}}{n !} G_{n}(x ; \omega)=2 \sum_{k \in \mathbb{Z}} \frac{e^{2 i \pi\left(k+\frac{1}{2}\right) x}}{\left(2 i \pi\left(k+\frac{1}{2}\right)-\log (\omega)\right)^{n}},
$$

for $0<x<1$ if $n=1$ and $0 \leq x \leq 1$ if $n \geq 2$. We note that

$$
E_{n}(x ; \omega)=-\frac{2}{n+1} B_{n+1}(x ;-\omega) \text { and } G_{n+1}(x ; \omega)=(n+1) E_{n}(x ; \omega) .
$$

Then we get immediately, from the Theorem 1, the following identities:

Theorem 3. Let $\omega \in \mathbb{C} \backslash\{0,-1\}$, and $x$ a real number. Then, for any $n \geq 1$ we have

$$
\begin{aligned}
& \frac{e^{2 i \pi x}}{(2 i \pi-\log (-\omega))^{n+1}}+\frac{e^{-2 i \pi x}}{(-2 i \pi-\log (-\omega))^{n+1}} \\
& =\sum_{m=1}^{\infty} \frac{\mu(m)}{m^{n+1}}\left(\frac{(-\omega)^{\frac{\{p x\}}{m}}}{2(n !)} E_{n}\left(\{m x\} ;-(-\omega)^{1 / m}\right)-\frac{m^{n+1}}{(-\log (-\omega))^{n+1}}\right),
\end{aligned}
$$

and for any $n \geq 2$ we have

$$
\begin{aligned}
& \frac{e^{2 i \pi x}}{(2 i \pi-\log (-\omega))^{n}}+\frac{e^{-2 i \pi x}}{(-2 i \pi-\log (-\omega))^{n}} \\
& \quad=\sum_{m=1}^{\infty} \frac{\mu(p)}{m^{n}}\left(\frac{(-\omega)^{\frac{\{m x\}}{m}}}{2(n !)} G_{n}\left(\{m x\} ;-(-\omega)^{1 / m}\right)-\frac{m^{n}}{(-\log (-\omega))^{n}}\right) .
\end{aligned}
$$

Remark 3. Note that we can write the last theorem in terms of $\log (\omega)$, thanks to the equalities $\log (-\omega)=\log \omega+i \pi$ if $-\pi \leq \arg \omega<0$ and $\log (-\omega)=\log \omega-i \pi$ if $0 \leq \arg \omega<\pi$.

\section{Apostol-Bernoulli numbers And applications}

4.1. Apostol-Bernoulli numbers. Let $n$ be an integer $\geq 1$ and $\omega \neq 1$. It is well known that

$$
B_{n}(x ; \omega)=\sum_{k=1}^{n}\left(\begin{array}{l}
n \\
k
\end{array}\right) B_{k}(0 ; \omega) x^{n-k}
$$

with

$$
B_{k}(0 ; \omega)=\frac{k}{\omega} \sum_{j=0}^{k-1}(-1)^{j} j ! S(k-1, j)\left(\frac{\omega}{\omega-1}\right)^{j+1} \text { for } k \geq 1 \text { (see [1]), }
$$

where $S(k, j)$ are Stirling numbers of the second kind defined by $S(k, j)=\frac{\Delta^{(j)} 0^{k}}{j !}$, with

$$
\Delta^{(j)} 0^{n}=\left(\Delta^{(j)} x^{n}\right)_{x=0}, \quad \Delta^{(j)} 0^{n}=0 \text { if } j>n, \quad \Delta^{(0)} 0^{0}=1,
$$

in the usual notation of finite differences. We call the numbers $B_{k}(0 ; \omega)$ the ApostolBernoulli numbers.

Now, we set $\omega=e^{2 i \pi z}$ with $z \notin \mathbb{Z}$. Then, for $k \geq 1$, we have

$$
B_{k}\left(0 ; e^{2 i \pi z}\right)=k \sum_{j=0}^{k-1}(-1)^{j} j ! S(k-1, j) e^{-2 i \pi z}\left(\frac{e^{2 i \pi z}}{e^{2 i \pi z}-1}\right)^{j+1} .
$$


On the other hand, an elementary computation allows us to write

$$
e^{-2 i \pi z}\left(\frac{e^{2 i \pi z}}{e^{2 i \pi z}-1}\right)^{j+1}=\frac{1}{(2 i)^{j+1}}\left(1+\cot (\pi z)^{2}\right)(i+\cot (\pi z))^{j-1} .
$$

Finally, we get the formula:

Theorem 4. For any integer $k \geq 1$, we have

$$
\begin{aligned}
& B_{k}\left(0 ; e^{2 i \pi z}\right) \\
& \quad=k \sum_{j=0}^{k-1}(-1)^{j} j ! S(k-1, j) \frac{1}{(2 i)^{j+1}}\left(1+\cot (\pi z)^{2}\right)(i+\cot (\pi z))^{j-1} .
\end{aligned}
$$

The formula (14) shows that, for $k \geq 1$, the $k^{\text {th }}$ Apostol-Bernoulli number $B_{k}\left(0 ; e^{2 i \pi z}\right)$ is a polynomial in terms of $\cot (\pi z)$ of degree $k$. Let us give the first values of $B_{k}\left(0 ; e^{2 i \pi z}\right)$ :

$$
\begin{aligned}
B_{1}\left(0 ; e^{2 i \pi z}\right)=\frac{1}{2 i}(\cot (\pi z)-i), B_{2}\left(0 ; e^{2 i \pi z}\right) & =\frac{1}{2}\left(1+\cot (\pi z)^{2}\right), B_{3}\left(0 ; e^{2 i \pi z}\right) \\
& =\frac{3 i}{4} \cot (\pi z)\left(1+\cot ^{2}(\pi z)\right) .
\end{aligned}
$$

From Theorem 2 and Theorem 4 , with $x=0$ and $\omega=e^{2 i \pi z}$, we obtain the following Möbius inversion formula:

Proposition 5. Let $n \geq 2$ be an integer and $z \notin \mathbb{Z}$ with $-1 / 2 \leq \Re(z)<1 / 2$. Then, we have

$$
\begin{aligned}
& \frac{z^{n}}{(1-z)^{n}}+\frac{(-z)^{n}}{(1+z)^{n}} \\
& \quad=-\sum_{m=1}^{\infty} \mu(m)\left(1+(\pi z / m)^{n} \sum_{j=1}^{n-1} A_{(n-1, j)}\left(\frac{d}{d z}(i+\cot )^{j}\right)(\pi z / m)\right)
\end{aligned}
$$

where $A_{(n, j)}=(-1)^{n-j} \frac{(-2 i)^{n-j} j !}{n !} S(n, j)$.

4.2. Euler's formulas for the zeta function at the even positive integers. Recall that the Riemann zeta function is given by

$$
\zeta(s)=\sum_{n=1}^{\infty} \frac{1}{n^{s}}, \quad(\Re(s)>1) .
$$

As an application of this section, we prove Euler's formulas for the zeta function at the even positive integers

$$
\zeta(2 k)=\frac{(-1)^{k+1} 2^{2 k-1}}{(2 k) !} \pi^{2 k} B_{2 k} .
$$

From the Proposition 5, we obtain for $n=2$ the formula

$$
\frac{z^{2}}{(1-z)^{2}}+\frac{z^{2}}{(1+z)^{2}}=-\sum_{m=1}^{\infty} \mu(m)\left(1+(\pi z / m)^{2} \cot ^{\prime}(\pi z / m)\right) .
$$

On the other hand, for $|z|<1$, we have the expansions

$$
\frac{z^{2}}{(1-z)^{2}}+\frac{z^{2}}{(1+z)^{2}}=2 \sum_{k=1}^{\infty}(2 k-1) z^{2 k}
$$


and

$$
z^{2} \cot ^{\prime}(z)=\sum_{k \geq 0}(2 k-1) \beta_{2 k} z^{2 k}, \quad \text { where } \quad \beta_{2 k}=(-1)^{k} \frac{2^{2 k}}{(2 k) !} B_{2 k} .
$$

Hence, from (18), after switching the order of summation in the double series, we have

$$
\begin{aligned}
& \sum_{m=1}^{\infty} \mu(m)\left(1+(\pi z / m)^{2} \cot ^{\prime}(\pi z / m)\right) \\
& =\sum_{k=1}^{\infty}(2 k-1) \pi^{2 k} \beta_{2 k}\left(\sum_{m=1}^{\infty} \frac{\mu(m)}{m^{2 k}}\right) z^{2 k} .
\end{aligned}
$$

Using the equalities (16), (17) and (19) we obtain

$$
2 \sum_{k=1}^{\infty}(2 k-1) z^{2 k}=-\sum_{k=1}^{\infty}(2 k-1) \pi^{2 k} \beta_{2 k}\left(\sum_{m=1}^{\infty} \frac{\mu(m)}{m^{2 k}}\right) z^{2 k} .
$$

By comparing the coefficients of $z^{2 k}$ in the right and left sides of formula (20), we obtain

$$
-2=\pi^{2 k} \beta_{2 k} \sum_{m=1}^{\infty} \frac{\mu(m)}{m^{2 k}} .
$$

Now, using the classical Möblius inversion

$$
\frac{1}{\zeta(s)}=\sum_{m=1}^{\infty} \frac{\mu(m)}{m^{s}}, \quad(\Re(s)>1),
$$

we recover, from (21) and (18), Euler's formulae (15).

\section{ACKNowledgments}

We are very grateful to the anonymous referee for many helpful comments and constructive remarks on a previous version of this work.

\section{REFERENCES}

1. T.M. Apostol, On the Lerch zeta function, Pacific J. Math. 1 (1951), 161-167. MR.0043843 $(13: 328 \mathrm{~b})$

2. A. Bayad, Fourier expansions for Apostol-Bernoulli, Apostol-Euler and Apostol-Genocchi polynomials, Math. Comp. 80 (2011), 2219-2221. MR.2813356

3. M. Benito, L.M. Navas and J.L. Varona, Möbius inversion formulas for flows of arithmetic semigroups, J. Number Theory 128 (2008), 390-412. MR2380327 (2009c:11160)

4. Q.-M. Luo, Fourier expansions and integral representations for the Apostol-Bernoulli and Apostol-Euler polynomials, Math. Comp. 78 (2009), 2193-2208. MR2521285(2010d:11029)

5. L.M. Navas, F.J. Ruiz and J.L. Varona, The Möbius inversion formula for Fourier series applied to Bernoulli and Euler polynomials, J. Approx. Theory 163 (2011), 22-40. MR.2741217 (2011j:11032)

DÉpartement de mathématiques, Université D'Evry VAl D'Essonne, BÂtiment I.B.G.B.I., 3̇̀me Étage, 23 Bd. De France, 91037 Evry Cedex, France

E-mail address: abayad@maths.univ-evry.fr

Département de mathématiques, Université d’Evry Val D’Essonne, BÂtiment I.B.G.B.I., 3̇̀me Étage, 23 Bd. De France, 91037 Evry Cedex, France

E-mail address: jchikhi@univ-evry.fr 